\title{
Effects of spin polarization on resonant photoemission from d-f states in $\mathrm{TbNi}_{2} \mathrm{Mn}_{\mathrm{x}}$ compounds
}

\author{
Vladimir I. Grebennikov ${ }^{1,2, *}$, Tatyana V. Kuznetsova ${ }^{1,2}$, Evgenii.G. Gerasimov ${ }^{1}$, and Nikolai.V. Mushnikov ${ }^{1}$ \\ ${ }^{1}$ M.N. Miheev Institute of Metal Physics UD RAS, 620990 Yekaterinburg, Russia \\ ${ }^{2}$ Ural Federal University, 620002 Yekaterinburg, Russia
}

\begin{abstract}
Resonant photoemission in narrow-band materials is described by the sum of first- and secondorder transitions, their quantum-mechanical interference leads to an increase in the spectrum from the valence bands and the appearance of an asymmetric dependence on the photon energy. These effects are studied theoretically and experimentally using the example of three-component intermetallic compounds $\mathrm{TbNi}_{2} \mathrm{Mn}_{\mathrm{x}}$. The competition between the elastic and inelastic photoemission channels leads to a different dependence of photoemission spectra from nickel and manganese on photon energy. The elastic channel is realized on atoms with large magnetic moments, the inelastic Auger decay occurs on atoms with small moments.
\end{abstract}

\section{Introduction}

Effects of superposition and interference of quantum states can be observed in resonance X-ray spectroscopy of solids. For the first time such effects were investigated in detail by Hugo Fano [1] in 1961 in connection with experiments on inelastic scattering of electrons by helium atoms. He developed a theory of a resonance with an asymmetric profile, called the Fano resonance, which arises as a result of interference of two wave processes. The nature of the interfering processes can be very different, in particular, the resonance was observed in the transmission and reflection spectra of photonic crystals. Let us turn to the process of photoemission of electrons from a solid with a narrow ( $3 d$ - or $4 f-$ ) band.

Left Fig. 1 shows the direct production of a photoelectron when a photon is absorbed in the valence band (VB). An additional channel is opened if the photon energy reaches an excitation edge for a core level. The photon first transfers the electron from the core level to the unoccupied state of the valence band (VB), and then the Coulomb interaction by

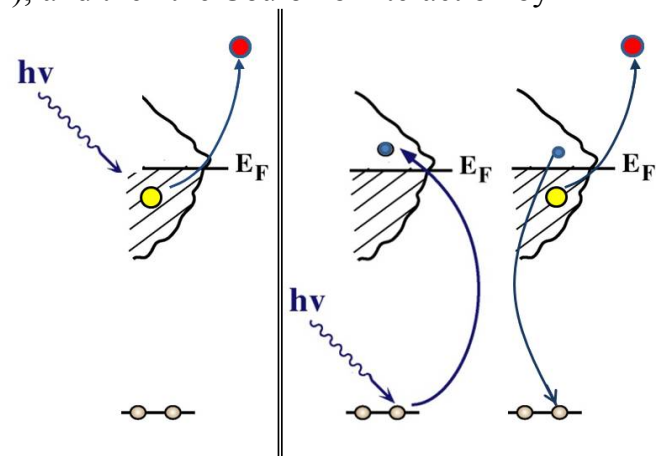

Fig. 1. Direct (left) and resonant (right) photoemission of electrons from a valence band. autoionization returns the excited electron back to the core level with the ejection of another VB electron to the free state. The same final state (photoelectron plus a hole in the valence band) is obtained as a result of two types of transitions.

\section{A theoretical background}

According to the principles of quantum mechanics, it is necessary to add the amplitudes of all such transitions and take the square of the modulus. As a result, the intensity of photoemission will be determined by the equality

$$
I\left(h f, E_{B}\right)=\sum_{k_{1}}\left|A+\sum_{k_{2}} \frac{M_{1} M_{2}}{h f-\left(E_{2}-E_{c}\right)-i g}\right|^{2} \delta\left(E_{B}-E_{1}\right)
$$

Here $A=\left\langle q|d| k_{1}\right\rangle$ is a matrix element (amplitude) of the electron dipole transition from the state $k_{1}$ with energy $E_{1}$ to the free state $q$ with the energy $E_{k i n}$ when the photon with energy $h f$ is absorbed. The same final state is the result of the second-order process whose amplitude is described by a fraction in (1): $M_{1}=\left\langle k_{2}|d| c\right\rangle$ is the dipole matrix element of the photon absorption, accompanied by the electron excitation from the core level $c$ to the free VB states $k_{2}$, the denominator is the difference of the corresponding energies, the imaginary additive $g$ stands for the half-width (the inverse lifetime) of the intermediate state. Then an autoionization Auger transition occurs due to the Coulomb interaction $M_{2}=\left\langle c q|Q| k_{2} k_{1}\right\rangle$, the electron $k_{2}$ returns to the core level $c$ with the emission of the valence electron $k_{1}$ into

* Corresponding author: vgrebennikov@list.ru 
the final (registered) state $q$. The process amplitude contains summation over all possible intermediate states $k_{2}$, and its squared modulus is summed over all finite states $k_{1}$. We note that there is also a contribution from the exchange matrix element $M_{2}{ }^{\prime}=\left\langle c q|Q| k_{1} k_{2}\right\rangle$. The delta function takes into account the energy conservation. The kinetic energy of photoelectrons and the energy of photons are measured experimentally, their difference has the meaning of the binding energy $E_{B}$ of the states $k_{1}$ from which the photoelectrons exit. Passing from summation over states to integration over energy (in the approximation of the weak energy dependence of the matrix elements), we write equation (1) in the form

$$
I\left(h f, E_{B}\right)=D\left(E_{B}\right)\left|A+M_{1} M_{2} G\left(h f+E_{c}-i g\right)\right|^{2} .
$$

Here $D(E)$ is the density of states (DOS), the integral $G(z)=\int d E_{1} D\left(E_{1}\right) /\left(z-E_{1}\right)$ is called the single-site Green function. We note that for a Lorentz DOS (width $\Gamma$ with center at $E_{0}$ ) the function has a simple form $G(z)=\left(z-E_{0}-i \Gamma\right)^{-1}$. For rectangular DOS (width 2u) $\quad G(z)=\left(\ln \left(z-E_{0}+\Delta\right)-\ln \left(z-E_{0}-\Delta\right)\right) / 2 \Delta$. The photoemission intensity as a function of the photon energy $h f$ and the binding energy of electrons $E_{B}$

$$
I\left(h f, E_{B}\right)=D\left(E_{B}\right)|A+B /(e-i)|^{2}, e=\left(h f-E_{\text {edge }}\right) / \Gamma
$$

is determined by the amplitudes of the first-order transition $A$ and the second order $B\left(=M_{1} M_{2} / \Gamma\right)$, the latter depending on the energy $e$ : its position is determined by the energy difference $E_{\text {edge }}=E_{0}-E_{c}$ of the unoccupied VB part and the core-level energy. The total width of $2 \Gamma$ includes the width of the core level and the width of the unoccupied part of the VB. In the approximation of the zero mean phase difference for the matrix elements of the first and second order, we obtain the equation

$$
I\left(e, E_{B}\right)=D\left(E_{B}\right)\left(A^{2}+\left(B^{2}+2 t A B e\right) /\left(e^{2}+1\right)\right),
$$

which includes modules $A$ and $B$ of the matrix elements, and the attenuation coefficient $t$ taking into account their phase fluctuations. Formula (4) describes the resonance enhancement of photoemission at the core-level excitation edge $(e=0)$. The interference of two transitions (the crossed term proportional to the energy e) generates an asymmetry of the profile with respect to photon energy. After normalization to the the first order intensity (or to the asymptotic value as e $\rightarrow \infty$ ), the equation (4) can be written as

$$
f(e)=1+\left(\mathrm{q}^{2}+2 t q e\right) /\left(e^{2}+1\right)
$$

where the parameter $q=B / A$ stands for the ratio of the second to first order matrix elements. It depends on the binding energy $E_{B}$ of the valence states and does not depend on the photon energy $e$. In the following we consider the profile of the function (5) with respect to the variable $e$ for fixed values of the photoelectron binding energy. The function $f(e)$ has a maximum and a minimum $f_{ \pm}=1+\left(q^{2} / 2\right)\left(1 \pm \sqrt{1+(2 t / q)^{2}}\right)$ at the points $e_{ \pm}=-(q / 2 t) \pm \sqrt{(q / 2 t)^{2}+1}$. The full width at half maximum of the function (the middle between maximum and minimum) is $e_{>}-e_{<}=2 \sqrt{(q / 2 t)^{2}+1}$. The asymmetry index $p$ is determined by the difference between the right and left half-widths of the spectrum divided by their sum (the full width)

$$
p=\frac{\left(e_{>}-e_{+}\right)-\left(e_{+}-e_{<}\right)}{e_{>}-e_{<}}=\frac{t}{q}\left(\frac{2}{1+\sqrt{1+(2 t / q)^{2}}}\right) \approx \frac{t}{q}(6)
$$

Here $e_{+}$is the energy of the maximum, $e_{>}$and $e_{<}$the right and left energy values at the half maximum. The asymmetry index (6) is reduced by a factor of $t$ compared with the Fano index 1/q [1].

\section{Experimental analyses}

Let us now analyze the experimental spectra. Fig. 2 shows the contour plot of the intensity of photoemission in the space of the electron binding energy and photon energy.
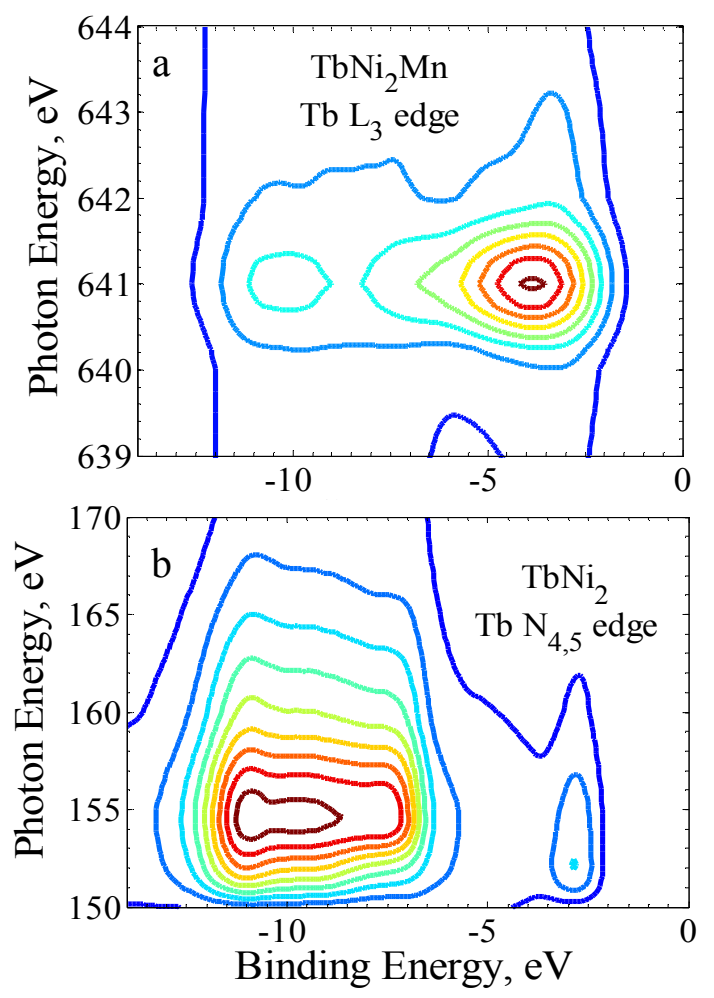

Fig. 2. The contour plot of the photoemission intensity near the $\mathrm{Mn} \mathrm{L}_{3}$ edge in the compound $\mathrm{TbNi}_{2} \mathrm{Mn}$ (a) and near $\mathrm{Tb} \mathrm{N}_{4.5}$ edges in the compound $\mathrm{TbNi}_{2}$ (b) The height of the maximum is divided into 10 levels with the same pitch.

A tenfold increase in the photoemission yield is observed at the $\mathrm{Mn} \mathrm{L}_{3}$ edge $(2 p \rightarrow 3 d$ transition $)$ in the intermetallic compound $\mathrm{TbNi}_{2} \mathrm{Mn}$. The width $\mathrm{Mn}$ of the spectrum with respect to the photon energy is $\sim 1.0 \mathrm{eV}$. The profile width at $\mathrm{Tb} \mathrm{N}_{4.5}$ edge (the transition $4 d \rightarrow \varepsilon f$, 
the so-called giant resonance, or shape resonance) is much larger $\sim 7.7 \mathrm{eV}$. The regions of localization of manganese state $2-6 \mathrm{eV}$ and terbium states $7-12 \mathrm{eV}$ are determined from the resonance enhancement. Fig. 3 shows the $\mathrm{Mn} \mathrm{L}_{2,3}$ and $\mathrm{Tb} \mathrm{N}_{4,5}$. x-ray absorption near edge structures (XANES). One can clearly see the strong multiplet splitting, which is very characteristic for transition and rare-earth elements compounds $[2,3]$.
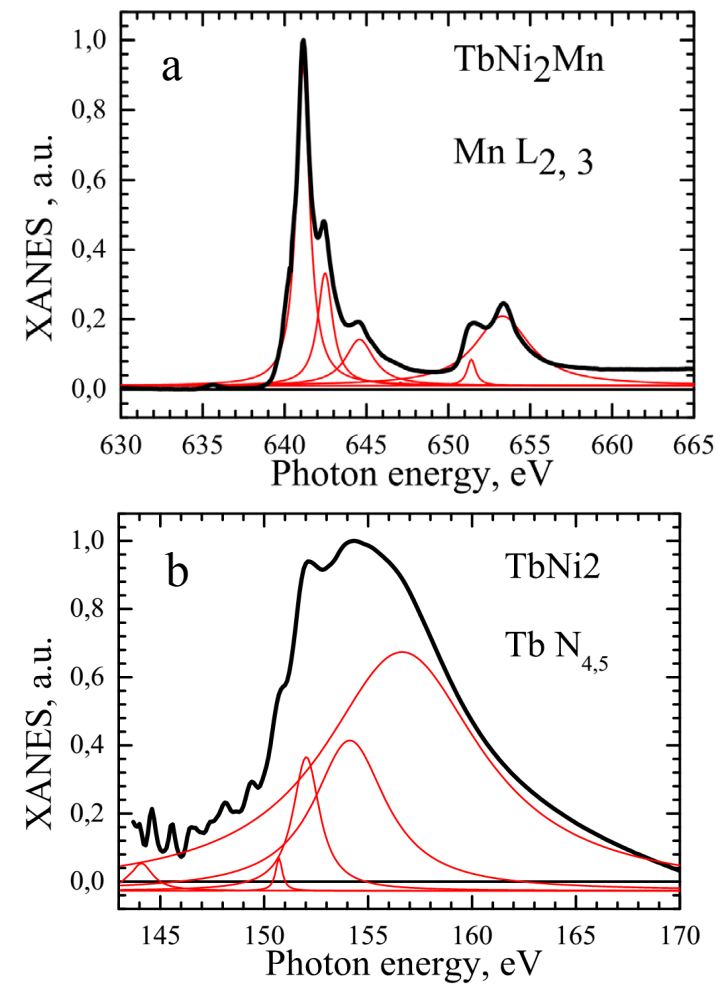

Fig. 3. X-ray absorption spectra at the $\mathrm{Mn}_{3}$ edge in $\mathrm{TbNi}_{2} \mathrm{Mn}$ (a) and on the $\mathrm{Tb} \mathrm{N}_{4.5}$ edge in $\mathrm{TbNi}_{2}$ (b). The decomposition of the curves into Lorentz peaks is shown.

Let us now consider the effects of the interference of the first and second order transitions as the photon energy changes. Formula (5) can be used for any binding energy $E_{B}$, but it is convenient to consider integrally two VB regions with binding energy $E_{B} 1-6 \mathrm{eV}$, formed predominantly to manganese (and nickel) states and a region of $7-12 \mathrm{eV}$ in which terbium states predominate. The experimental photoemission intensity on the $\mathrm{Mn} \mathrm{L}_{3}$ edge in $\mathrm{TbNi}_{2} \mathrm{Mn}$ (a) and on $\mathrm{Tb} \mathrm{N}_{4.5}$ edge in $\mathrm{TbNi}_{2}$ (b) averaged over these intervals is shown by the dots in Fig. 4 as a function of the energy of the exciting photon energy $h f$. The solid lines show the results of fitting according to the formula (5) with three fitting parameters $\Gamma\left(0.48\right.$ for $\mathrm{TbNi}_{2} \mathrm{Mn}$ (panel $a$ ) and $3.60 \mathrm{eV}$ for $\mathrm{TbNi}_{2}$ (panel $b)), q(2.78$ for $1-6 \mathrm{eV}$ and 1.07 for $7-12 \mathrm{eV}$, curves $a$ and 1.82 for $1-6 \mathrm{eV}$ and 3.59 for $7-12 \mathrm{eV}$, curves $b$ ) and $t$ (see the values below). Our simple model gives a good description of profiles on the excitation edges of manganese and terbium.

Note that the resonance on manganese not only leads to an increase in the electron yield from the manganese bands, but also more than doubles the photoemission from the terbium states of $7-12 \mathrm{eV}$ (which seem to be on the side). In turn, the resonance in terbium leads to a threefold increase in the photoemission yield from the nickel band $(1-6 \mathrm{eV})$. There is a strong interaction and competition between transitional and rare-earth components.
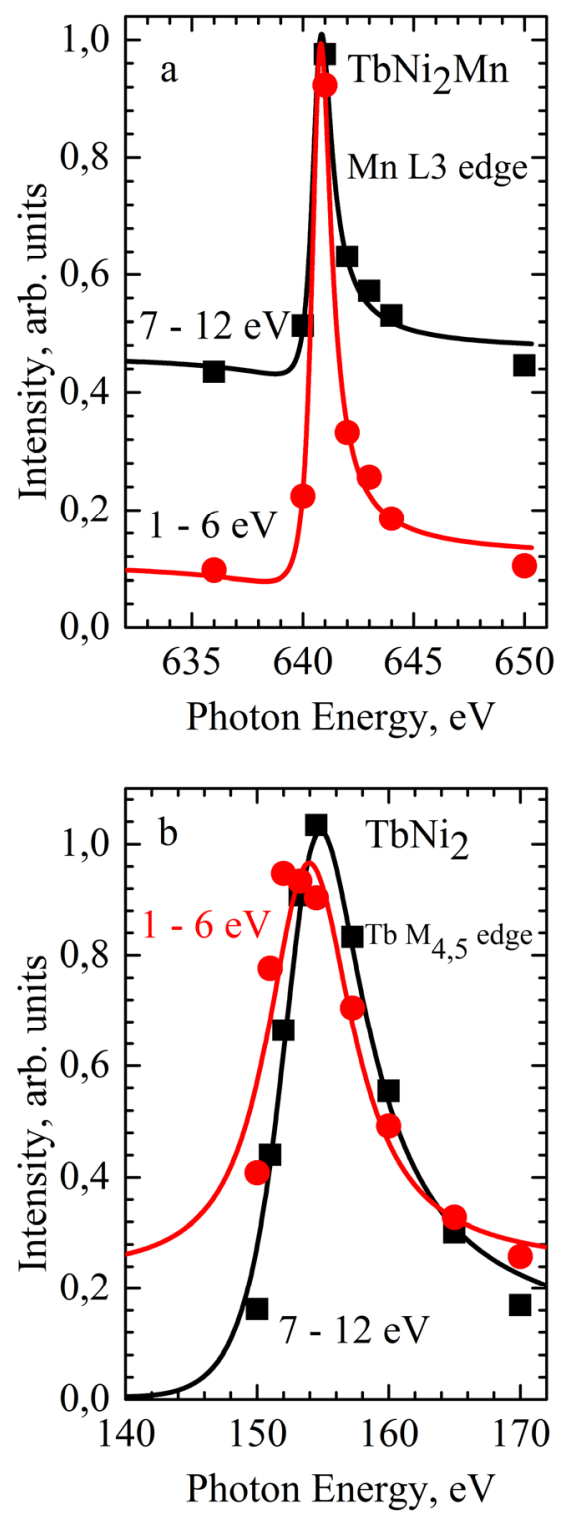

Fig. 4. The experimental photoemission intensities in the binding energy range $7-12 \mathrm{eV}$ (black squares) and $1-6 \mathrm{eV}$ (red dots) on the $\mathrm{Mn} \mathrm{L}_{3}$ edge in $\mathrm{TbNi}_{2} \mathrm{Mn}$ (a) and on $\mathrm{Tb} \mathrm{N}_{4.5}$ edge in $\mathrm{TbNi}_{2}$ (b). The lines show a fit according to Eq. (5).

The second-order photoemission process (with allowance for the resonant denominator) is much more efficient than the direct photoelectric effect from the valence band at both edges. The point is that the photoionization cross section (the Fourier transform of the wave function) is small for valence states weakly localized in space. It is much larger for strongly localized wave functions of the core levels. The Coulomb interaction acting in the second stage is also stronger than the interaction with radiation. The evidence of interference is the asymmetry of the profiles, which is seen directly in Fig. 4. Fig. 5 shows the values of the asymmetry index $p$. They are large in terbium and manganese $(a)$ and much less on nickel $(1-6 \mathrm{eV}$ band, panel $b$ ). 
The correlation of the two transitions $t$ is given below

$$
\begin{array}{cccc}
\mathrm{Mn} \mathrm{L}_{3}(\mathrm{~Tb}) & \mathrm{Mn} \mathrm{L}_{3}(\mathrm{Mn}+\mathrm{Ni}) & \mathrm{Tb} \mathrm{N}_{4,5}(\mathrm{~Tb}) & \mathrm{Tb} \mathrm{N}_{4,5}(\mathrm{Ni}) \\
0.29 & 0.58 & 1 & 0.09
\end{array}
$$

It is equal to 1 only for terbium resonance in the range of terbium states. In other cases it is much less. The reason here is not only in the probable accidental phase failure of the intermediate state wave function, but also the states obtained as a result of the transitions of the first and second order can be somewhat different. Auger autoionization involves overlapping the wave functions of the valence and core states, but direct photoemission, of course, does not. This difference leads only to partial interference.

The resonance emission not always follows the elastic scenario described in Fig. 1. Now we consider what processes occur in the same compound on the nickel atom whose resonant XPS is shown in Fig. 6 . Again, the resonance multiple amplification of the photoemission yield from the valence states is observed at the $\mathrm{Ni} \mathrm{L}_{3}$ edge $(h f=854 \mathrm{eV})$. At the same time, the spectrum shape changes greatly above the edge. The maximum shifts evenly along the binding energy scale (and it is stationary in the kinetic photoelectron energy scale). This is a typical LVV Auger line. The final state contains two holes in the valence band and an excited electron above the Fermi level, which carries away the excess photon energy. It should be noted that in the region of the Auger line generation the resonant photoemission spectra provide detailed information for the investigation of the two-hole correlation effects [4].

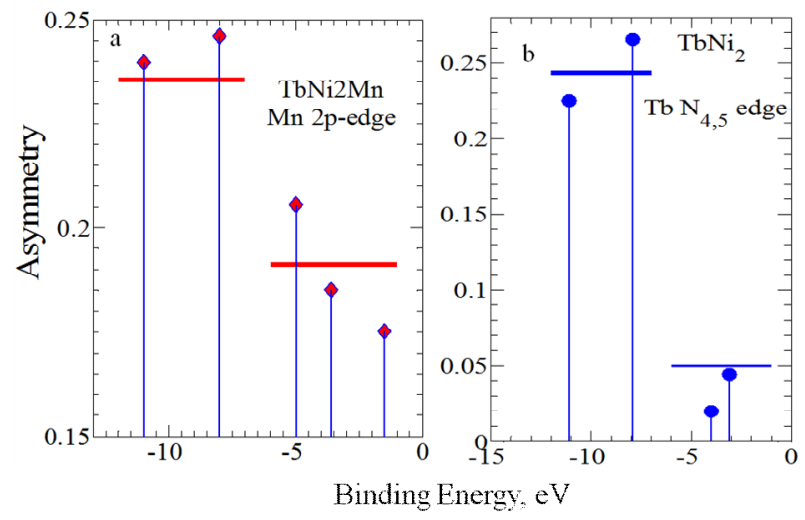

Fig. 5. The asymmetry index of profiles at different binding energies (points). The horizontal lines show mean values in the intervals $1-6$ and $7-12 \mathrm{eV}$ at the excitation edges of manganese (a) and terbium (b).

Thus, there is a competition between the elastic channel of virtual excitation of the core level, as described above, and the real (energy conservation in the intermediate state) Auger process in which the $2 p$ hole is occupied by one valence electron, whereas another valence electron leaves the sample and it is recorded by the detector. The first process is dominant in case of the minganise atoms, and the second process prevails for the nickel atoms. This is closely related to the spin magnetic state of the atoms $[5,6]$, as it is easily seen from a simple model. Let the manganise atom have five $d$ electrons. According to Hund's rule, they all have the same spin projections $\uparrow$, and they completely fill the corresponding spin subband. As a result, only the electron with the spin $\downarrow$ takes part in the $2 \mathrm{p} \rightarrow 3 \mathrm{~d}$ transition due to spin conservation. Consequently, only one electron from the six $d$ electrons can occupy the $2 p$ hole (with the spin $\downarrow$ ), and this is the electron occupied the $d$ state due to the photon absorption, i.e. in this case, the elastic resonant photoemission channel is implemented. If now the ground state has 8 electrons in the $(5 \uparrow 3 \downarrow)$, configuration, then again, a hole with the spin down arises, and the $\mathrm{L} \downarrow(5 \uparrow 3 \downarrow 1 * \downarrow)$ excited state is obtained. Now, 4 VB electrons $\downarrow$ can occupy the $\mathrm{L} \downarrow$ photohole. Hence, with a decrease in the spin polarization (occupation of the $3 d$ band), the probability of the elastic channel decreases, and the contribution of the inelastic Auger channel of electron emission increases.

Thus, resonant photoemission spectroscopy has a selective sensitivity to the components of compounds and makes it possible to measure the fine coherence effects in materials.

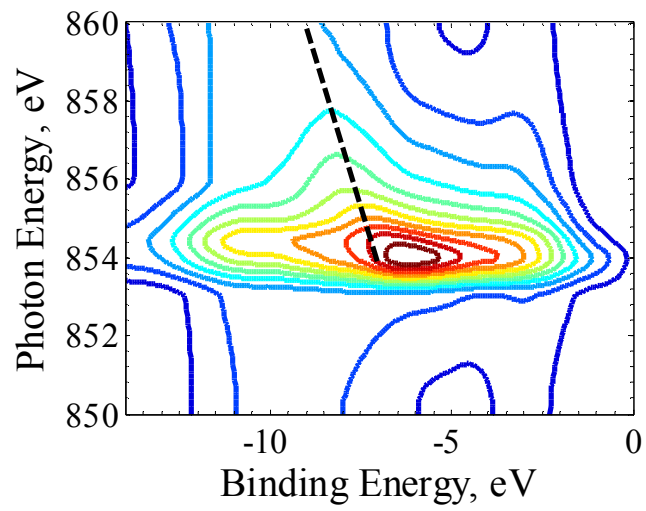

Fig. 6 The contour plot of the photoemission intensity near the $\mathrm{Ni} \mathrm{L}_{3}$ edge in $\mathrm{TbNi}_{2} \mathrm{Mn}$.

The research was carried out within the state assignment of FASO of Russia (theme "Quant" No. 01201463332), supported in part by RFBR (No. 18-02-00060) and UD RAS (No.15-8-210). The authors express their gratitude to A. Preobrazhenskii and N. Vinogradov (synchrotron MAX-Lab, Lund, Sweden) for their assistance in carrying out experiments.

\section{References}

1. U. Fano, Phys.Rev.. 124, 1866 (1961)

2. T. V. Kuznetsova, V. I. Grebennikov, N. V. Mushnikov, E. G. Gerasimov, A. Buling, C. Derks, M. Neumann, Bull. RAS: Physics, 77, 226 (2013)

3. V.I. Grebennikov, T.V. Kuznetsova, A.G. Kuchin, Bull. RAS: Physics. 79, 134 (2015)

4. V. I. Grebennikov, T. V. Kuznetsova, and M. V. Yakushev, Bull. Russ. Acad. Sci.: Phys., 77, 1123 (2013)

5. M. V. Yablonskikh, Yu. M. Yarmoshenko, V. I. Grebennikov, E. Z. Kurmaev, S. M. Butorin, L.-C. Duda, J. Nordgren, S. Plogmann, and M. Neumann, Phys. Rev. B, 63, 235117 (2001)

6. V. I. Grebennikov, Poverkhnost, 11, 41 (2002) 